Increasing false recognition rates with confirmatory feedback:

\title{
A phenomenological analysis
}

Peter Frost, Donna Lacroix, Nicole Sanborn

Southern New Hampshire University

Citation: Frost, R J., Lacroix, D., \& Sanborn, N. (2004). Increasing false recognition rates with confirmatory feedback: A phenomenological analysis. The American Journal of Psychology, 116(4), 515-525. http://www.jstor.org/stable/1423658

Received for publication: August 26, 2002. Revision received: February 11, 2003 


\begin{abstract}
During a simulated witness interrogation, participants were encouraged to confabulate an account consistent with false information concerning a videotaped event. The interviewer verbally affirmed some false responses. Previous research has shown that, a week later, participants often recognize confabulated events that were affirmed by the experimenter as being from the video. What is unclear is whether confirmatory feedback encouraged a change in the mental representation of the confabulated events to fit the original event or confirmation might have merely encouraged a change in beliefs about the event. To further understand the mechanisms that underlie the confirmatory feedback effect, participants were asked to judge the phenomenological experience associated with false recognition.
\end{abstract}


Increasing false recognition rates with confirmatory feedback 3

\section{Increasing false recognition rates with confirmatory feedback: A phenomenological analysis}

Extensive scientific literature over the past four decades has demonstrated that exposure to misinformation can lead to false memories, defined as false beliefs about the past that are experienced as memories (Belli, Lindsay, Gales, \& McCarthy, 1994, Ceci, Huffman, \& Smith, 1994; Frost, 2000; Heaps \& Nash, 2001; Loftus, Miller, \& Burns, 1978; Zaragoza \& Koshmider, 1989). Applied research has demonstrated that bias, as expressed by therapists (Loftus \& Ketcham, 1994) and forensic interrogators (Zaragoza, Payment, Ackil, Drivdahl, \& Beck, 2001), can lead to false memories in the interviewee.

For example, interviewers might express their biases through feedback to shape witnesses' testimony, either intentionally or unintentionally. They might reinforce the retrieval of memories that are consistent with their beliefs and ignore accounts that are inconsistent with their beliefs. Interviewers sometimes pressure witnesses to provide accounts about events that the interviewer believes to have occurred, even when the witness did not see the suggested events (Leo, 1996; Zaragoza et al., 2001).

Zaragoza et al. (2001) assessed whether providing confirmatory feedback after participants knowingly confabulated (made up) an eyewitness account of an event would later increase false memory of the forced confabulations. Adult participants first watched a videotaped excerpt - the simulated eyewitness event - then engaged in interviews during which they were forced to answer questions that required a confabulated response about events depicted in the videotaped excerpt. For example, participants were asked, "Where was Delany bleeding?” when, in fact, he was not shown bleeding in the videotaped excerpt. The interviewer also reinforced some confabulated responses with confirmatory feedback (e.g., "Yes, is the correct answer”) and provided neutral feedback for the remaining confabulated responses (e.g., "OK "). A week later, participants answered a recognition test that included questions about details they had confabulated during the interview. Participants were also asked to rate their confidence in each of their answers. Participants recognized confabulated events as being from the videotaped event, even after they were warned that some questions during the prior interview referred to events that never happened. Furthermore, confirmatory feedback increased the frequency of false memory significantly more than neutral feedback. Confirmatory feedback 
was also associated with an increase in confidence ratings. Zaragoza et al. (2001) concluded that social influence can be a potent catalyst to the creation of false memory.

What is unclear are the mechanisms that underlie the confirmatory feedback effect. Why are participants more likely to recognize false information that they had knowingly confabulated in the past when others confirmed the confabulation?

One possibility is that the mental representation of the confabulated incident changes after confirmation by others. Zaragoza et al. (2001) suggested that confirmation might encourage participants to reflectively elaborate the confabulated event in an effort to make it fit with the events they witnessed. It has been documented that discrimination between imagination and reality can be challenging, especially when imagined events contain many features that are typical of perceived events (Johnson, Foley, \& Leach, 1988). Drivdahl and Zaragoza (2001) point out that imagining an event involves reflectively elaborating on an idea, making it more like real memories by general (e.g., increasing familiarity) and specific (e.g., adding what seem like sensory and perceptual details) mechanisms. Although some studies (Drivdahl \& Zaragoza, 2001; Thomas \& Loftus, 2002) have demonstrated that people are more susceptible to false memories after imagining false information, it is unclear whether this mechanism contributes to the confirmatory feedback effect.

Another potential reason for the confirmatory feedback effect is that participants believe an event was seen because it was previously confirmed, without necessarily experiencing a change in the mental representation of the event. In other words, the confirmatory feedback effect might induce a belief that a false event occurred without the conscious experiences and images that are often associated with the original event, as often occurs with real memory. Any doubts the participants might have had initially about a confabulated event might have been dispelled after the experimenter confirmed the event. Indeed, Zaragoza et al. (2001) found that confirmatory feedback eliminated the negative correlation found between verbal resistance to confabulations (e.g., as measured by the number of verbalized disagreements with the question) and the rate of false recognition. This hypothesis suggests that affirmation of false events by the interviewer does not strengthen false memories per se but merely changes beliefs about false event details. Although participants in the study by Zaragoza et al. (2001) were warned about the potential for misinformation during the interview, they may have already decided which items occurred, and they might have forgotten the source of their decisions. 
To examine these potential mechanisms, this study focused on the phenomenology-the perceived subjective quality — of previously confirmed false memories. Participants were asked to judge recognized events as remembered or known. A remembered event is characterized as an episodic recollection, including the awareness of aspects of the encoding event such as images, thoughts, feelings, and knowledge specific to the event (Heaps \& Nash, 2001; Tulving, 1985). Tulving (2002) also emphasizes that remembered events are associated with an awareness of the time and place specific to the original event. If a memory is judged as known, then the event is believed to have occurred during the eyewitness episode, but it lacks the imagery of the moment (Tulving, 2002).

Does confirmatory feedback lead to a greater number of remember or know judgments associated with false events? The answer to this question can be informative in regard to the mechanisms responsible for the confirmatory feedback effect suggested earlier. For instance, if participants tend to recognize confabulated events more often after confirmatory feedback merely because they believed the event must have occurred given the feedback, then we would expect more know judgments to be associated with reinforced false memories. However, if participants recognize confabulated events more often after confirmatory feedback because the confirmation leads them to embellish the event with more imagery and false details, then we would expect more remember judgments to accompany retrieval of reinforced false events.

\section{EXPERIMENT}

\section{METHOD}

\section{Participants}

The experiment included 40 undergraduate students from Southern New Hampshire University who were recruited on a voluntary basis. Fifteen students participated in a free group, and the $25^{1}$ remaining students participated in a forced group (see an explanation of free and

\footnotetext{
${ }^{1}$ Sixteen participants were originally assigned to the free group and 26 participants were
} originally assigned to the forced group. However, one participant was dropped from each group because of incomplete data. 
Increasing false recognition rates with confirmatory feedback 6

forced groups under Procedure and Materials). Participants were randomly assigned to each group.

\section{Procedure and materials}

The procedures in this study were similar to those used by Zaragoza et al. (2001). However, some exceptions are noted here.

Students participated in pairs. After signing consent forms, participants viewed a 15-min excerpt from the 1985 movie Clue (by Paramount Pictures). The excerpt depicts a mystery in which a man is murdered at a dinner party held at a mansion. Many of the people gathered at the mansion had been blackmailed by the victim.

Immediately after viewing the videotaped excerpt, participants were separated and interviewed by different experimenters. Participants were randomly assigned to a free group or forced group. Before the interview began, participants in the forced group were told they must

provide an answer to every question and were explicitly instructed to guess if they did not know an answer. Participants in the free group were explicitly instructed to respond only to the questions they could answer without guessing. The purpose of the free group was to verify that participants did not spontaneously answer the false event questions.

During the interview, each participant responded to 12 questions about the movie excerpt after answering 2 questions about whether they had seen the movie Clue before and, if so, how long ago they had seen it. Eight of the questions were true event questions about events from the videotaped excerpt. The four remaining questions were false event questions requiring the participant to confabulate answers to questions about events that never occurred in the video presentation. For example, in discussing a scene from the video, the experimenter asked, "How many guests did it take to force open the locked door to the room with the screaming maid?" To answer this question, participants had to confabulate a response because although the guests did attempt to push the door open, it was only when the maid unlocked the door that the guests entered. If participants in the forced group resisted answering the question, the experimenter prompted an answer by asking participants to provide their best guess. Two sets of four false event questions were provided in each session, one set for one of the participants in the pair and the other set for the other participant in the pair. The same number of participants was exposed to each of the eight false event questions. 
The forced group received feedback for their responses during the interview. Each participant received confirmatory feedback (e.g., “That's right, [four] is the correct answer”) after confabulated responses to two of the false event questions. The two remaining confabulated responses were followed by neutral feedback delivered with flat affect (e.g., “[four], OK”). To counterbalance, each false event question served equally often in the confirmatory and neutral feedback conditions. All responses to true event questions were followed by neutral feedback.

After 1 week, recognition of false events was assessed by a yes-no test constructed in a format similar to that of previous research on forced confabulation (Ackil \& Zaragoza, 1998; Zaragoza et al., 2001). During the second session, participants were warned that the experimenter in the first session made some mistakes and asked them questions about events that never occurred in the video. Participants were told to answer yes-no questions based only on what they remembered from the video. These instructions were provided to avoid social pressure to respond consistently across test sessions.

Twenty yes-no questions were asked in the following form: "When you watched the video, did you see___ ? " For example, the question about the false event involving the forcing open of a locked door was presented as, "During the video, did you see the guests force open the locked door to the room with the screaming maid? YES OR NO.” The test included 4 questions about false event items the participant confabulated during the interview (with 2 items followed by confirmatory feedback during the interview and 2 items followed by neutral feedback during the interview), 4 questions about the set of false event items the participant's partner confabulated during the interview (to serve as a base rate), and 12 filler questions that included questions based on 5 true event questions from the interview, 4 additional items from the video, and 3 new items. All types of questions were presented in random order.

The words "remember" and "know" followed each question in the yes-no recognition test. Participants were asked to circle the word that best characterized their experience recognizing the item in question based on the following instructions read to them before the yesno test was administered:

For each yes response to the following questions about the video you saw a week ago, please circle either the word, remember or know. Choose the word that best characterizes your experience recognizing the items referred to in each question. A remember judgment means that your memory of the item in question includes 
awareness of aspects of the encoding environment, such as the images, feelings, and thoughts that accompanied the item when you originally studied it. A remembered item is vivid and remembered with pictorial imagery characteristic of how it originally looked in the video. A know judgment means that you believe an item was seen in the video, but memory for the item is not vivid and lacks imagery and context.

To help ensure that participants understood these instructions, they were asked whether they had any questions. In addition, these instructions were displayed on an overhead projector while they responded to the yes-no recognition test.

Although the procedures of this study are similar to those of Zaragoza et al. (2001), there is an important distinction. During the recognition test participants were not tested on their memory of their confabulated response (e.g., the number of guests), as they were in Zaragoza et al. (2001). Participants in the present study were asked about the presupposed false event. Because participants were given false information about a single embedded detail in the question, they might not have knowingly confabulated answers. Future research could examine whether there is a difference in the effects of feedback on phenomenal experiences when centrally presented information is used (as in Zaragoza et al., 2001) and when it is incidental or a precondition for some centrally presented information (as in this study).

\section{RESULTS}

First, it was important to determine whether participants had seen the movie Clue before the experiment. Most participants $(80 \%)$ indicated that they had not seen the movie Clue before seeing the excerpt during the experiment. The remaining $20 \%$ of students indicated that they saw the movie 5 years ago on average. Because the analyses for participants who had and had not previously seen the movie Clue led to the same results, these groups were combined for the following analyses.

Data from the interview were also analyzed to determine whether participants were truly forced to confabulate during the interview. This was important because answers to false event questions might have been spontaneous, independent of the forced group procedures. However, none of the participants in the free group spontaneously provided answers to false event questions. Therefore, it is unlikely that responses to false event questions in the forced-group 
condition were merely spontaneous. Moreover, forced-group participants overtly resisted answering false event questions $25 \%$ of the time, an indication that they felt forced to respond despite the instructions to provide an answer even if they had to guess. In contrast, forced-group participants overtly resisted answering true event questions $4 \%$ of the time.

Consistent with previous research (Zaragoza et al., 2001), participants in the present study recognized false event items associated with forced confabulation, especially when these items were confirmed by the experimenters during the interview. The proportion of "yes" responses to false event questions was greater than the base rate for false event questions (.19), for both neutral (.38) and confirmatory (.53) feedback conditions, $F(2,48)=8.81, p<.01$. Consistent with the confirmatory feedback hypothesis, recognition of false event items associated with confirmatory feedback did exceed that for neutral feedback, $t(24)=1.98, p<.05$.

The proportions of remember and know judgments shown as a function of recognition question type are listed in Table 1. As is typical of delayed tests (Gardiner \& Java, 1991; Roediger, Jacoby, \& McDermott, 1996), the proportion of remember judgments associated with true event details was slightly lower than the proportion of know judgments.

Table 1. Proportion of "yes" responses as a function of question type, broken down by the proportion of remember and know judgments

\begin{tabular}{|c|c|c|c|}
\hline Question type & $\begin{array}{l}\text { Proportion of } \\
\text { "yes" responses }\end{array}$ & Remember & Know \\
\hline False event, confirmatory feedback & .53 & .15 & .38 \\
\hline False event, neutral feedback & .38 & .17 & .21 \\
\hline False event, base rate & .19 & .05 & .13 \\
\hline True event & .70 & .34 & .36 \\
\hline
\end{tabular}

Know judgments exceeded remember judgments to an even greater extent when false event items were recognized.

Of primary importance to the present study was the pattern of remember or know judgments associated with different kinds of feedback to false event items. There was no significant difference between the proportion of false event items followed by a "yes" response and a remember judgment in the confirmatory (.15) and neutral (.17) feedback conditions. The proportion of false event items followed by a "yes" response and a know judgment was higher for the confirmatory feedback condition (.38) than for the neutral feedback condition $(.21), t(24)$ 
Increasing false recognition rates with confirmatory feedback 10

$=1.72, p<.05$. Overall, the type of feedback influenced the proportion of "yes" responses and know judgments but had no influence on the proportion of "yes” responses and remember judgments. This finding supports the hypothesis that participants were more likely to recognize false event items that had previously been confirmed because of a strengthening of beliefs in the false event rather than an increase in the vividness of the mental representation of the recognized false event caused by embellishment or other processes.

\section{DISCUSSION}

This study reveals that the confirmatory feedback effect for false event recognition probably is not caused by false memory. Genuine false memories are associated with recollective experiences, or awareness of the episodic qualities of the original event (Heaps \& Nash, 2001). Researchers (e.g., Zaragoza et al., 2001) have claimed that confirmatory feedback increases false memories. Yet the findings of this study suggest an alternative explanation. False event recognition, after being confirmed by the experimenter, was often judged as known rather than remembered. Had confirmatory feedback enhanced the remember experiences associated with false event information, then we would expect the opposite: more false recognition, confirmed by the experimenter, being accompanied by remember judgments. Instead, there was actually a greater likelihood of recognized false events being judged as known when confirmatory feedback was used rather than neutral feedback.

Therefore, it is more likely that confirmatory feedback strengthens beliefs about the validity of false event details without necessarily facilitating the formation of false memory. Consistent with this finding, Zaragoza et al. (2001) found that confirmatory feedback tends to increase the confidence that participants have in falsely recognized events. Although confirmatory feedback strengthens belief and confidence in false events, it does not necessarily alter the conscious recollection associated with it as indicated by remember judgments.

Perhaps remembering was defined narrowly in this study. Although the instructions to participants indicating that remember judgments are associated with an awareness of aspects of the encoding events such as images, thoughts, and feelings specific to the original event are consistent with those of past studies (Heaps \& Nash, 2001; Tulving, 1985), not all researchers specify that remember judgments should be vivid. This definition might have limited the number of remember judgments, perhaps accounting for why remember and know judgments were equal 
for true events. However, the recognition test was administered after a 1-week retention interval, which often leads to less remember judgments and more know judgments, as shown in prior research using similar intervals (Roediger et al., 1996; Gardiner \& Java, 1991). Furthermore, because the same instructions were associated with each feedback condition (i.e., feedback was manipulated within participants), it is unlikely that the instructions biased against remember judgments more in one feedback condition than in another.

Although the evidence from this study indicates that participants changed their beliefs about the false event information, were they aware that feedback by the experimenters swayed their belief in the false event information? Despite being warned that some information revealed by the interviewer was incorrect, participants still were more likely to retrieve false event information associated with confirmatory rather than neutral feedback. Had they been aware of which details were selected based on belief encouraged by a discredited interviewer, then the confirmatory feedback effect should have been eliminated or weakened, but it was not.

The finding that participants can distinguish recognition of false events from that of true events with remember or know judgments is consistent with recent research (Anastasi, Rhodes, $\&$ Burns, 2000; Frost, 2000). For example, Frost (2000) showed that when participants read about misinformation that contradicts events seen in an eyewitness episode, then misinformation is more likely to be judged as known rather than remembered after short (10 min) and long delays $\left(1\right.$ week). ${ }^{2}$ However, these studies did not involve confabulation or the confirmatory feedback paradigm.

In summary, this study produced three important findings. First, it confirmed the hypothesis that confabulating about a false event can lead to recognition of the false event. Second, the results showed that confirmatory feedback by an interviewer can increase the odds that a witness will falsely recognize confabulated events. Third, this study revealed that recognition of false events tended to be judged as known, especially when accompanied by confirmatory feedback as opposed to neutral feedback. This finding suggests that the confirmatory feedback effect occurs mainly because of a sway in belief rather than the

\footnotetext{
${ }^{2}$ When misinformation did not contradict what was seen in the original eyewitness
} episode, retrieval of misinformation was more likely to be judged as remembered after long delays. 
elaboration or enhanced vividness of a false memory. This finding also suggests that witnesses can distinguish true and false recognition via remember or know judgments to some extent.

The present study suggests some avenues for future study. For example, why does confirmatory feedback increase the proportion of know judgments? Perhaps participants select know judgments because of a phenomenological sense of familiarity that is made more salient when the false event item was followed by confirmatory feedback. In other words, are know judgments associated with confirmed false events merely a response bias based on a sense of familiarity?

The current findings have important practical implications. Efforts must be directed at making feedback during eyewitness interrogations as neutral as possible. This study revealed that statements as plain as "that's correct" can increase false recognition. A simple affirmation can make the difference between recognition and no recognition of some aspect of an eyewitness event.

\section{Notes}

Several students helped conduct this study and analyze the data. We would like to thank Christine Hatch, Lauren Sharron, Amber Snedeker, and Tristin Turner for their contributions. We are also grateful for the insights and suggestions provided by Dr. Donelson Dulany and Dr. Jeffrey Anastasi. This study was presented as a paper at the 42nd annual meeting of the New England Psychological Association, Nashua, New Hampshire, October 2002.

Correspondence about this article should be addressed to Peter Frost, Department of Psychology, Southern New Hampshire University, 2500 North River Road, Manchester, NH 03106-1045 (e-mail: p.frost@snhu.edu). Received for publication August 26, 2002; revision received February 11, 2003.

\section{References}

Ackil, J. K., \& Zaragoza, M. S. (1998). Memorial consequences of forced confabulation: Age differences in susceptibility to false memories. Developmental Psychology, 34, 13581372. 
Anastasi, J. S., Rhodes, M. G., \& Burns, M. C. (2000). Distinguishing between memory illusions and actual memories using phenomenological measurements and explicit warnings. American Journal of Psychology, 113(1), 1-26.

Belli, R. R, Lindsay, D. S., Gales, M. S., \& McCarthy, T. T. (1994). Memory impairment and source misattribution in postevent misinformation experiments with short retention intervals. Memory \& Cognition, 22(1), 40-54.

Ceci, S. J., Huffman, M. L., \& Smith, E. (1994). Repeatedly thinking about a non-event: Source misattributions among preschoolers. Consciousness and Cognition, 3, 388-407.

Drivdahl, S. B., \& Zaragoza, M. S. (2001). The role of perceptual elaboration and individual differences in the creation of false memories for suggested events. Applied Cognitive Psychology, 15, 265-281.

Frost, R J. (2000). The quality of false memory over time: Is memory for misinformation “remembered” or “known”? Psychonomic Bulletin \& Review, 7(3), 531-536.

Gardiner, J. M., \& Java, R. I. (1991). Forgetting in recognition memory with and without recollective experience. Memory \& Cognition, 19, 617-623.

Heaps, C. M., \& Nash, M. (2001). Comparing recollective experience in true and false autobiographical memories, Journal of Experimental Psychology: Learning, Memory, and Cognition, 27(4), 920-930.

Johnson, M. K., Foley, M. A., \& Leach, K. (1988). The consequence for memory of imagining in another person's voice. Memory \& Cognition, 16, 337-342.

Leo, R. A. (1996). Inside the interrogation room. Journal of Criminal Law and Criminology, 86, 266-303.

Loftus, E. R, \& Ketcham, K. (1994). The myth of repressed memory: False memories and allegations of sexual abuse. New York: St. Martin's Griffin.

Loftus, E. R, Miller, D. G., \& Burns, H. J. (1978). Semantic integration of verbal information into a visual memory. Journal of Experimental Psychology: Human Learning and Memory, 4, 19-31.

Roediger, H. L. III, Jacoby, J. D., \& McDermott, K. B. (1996). Misinformation effects in recall: Creating false memories through repeated retrieval. Journal of Memory and Language, $35,300-318$. 
Thomas, A. K., \& Loftus, E. F. (2002). Creating bizarre false memories through imagination. Memory \& Cognition, 30(3), 423-431.

Tulving, E. (1985). Memory and consciousness. Canadian Psychologist, 26, 1-12.

Tulving, E. (2002). Episodic memory: From mind to brain. Annual Review of Psychology, 53(1), $1-25$.

Zaragoza, M. S., \& Koshmider, J. W. III. (1989). Misled subjects may know more than their performance implies. Journal of Experimental Psychology: Learning, Memory, and Cognition, 15(2), 246-255.

Zaragoza, M. S., Payment, K. E., Ackil, J. K., Drivdahl, S. B., \& Beck, M. (2001). Interviewing witnesses: Forced confabulation and confirmatory feedback increase false memories.

Psychological Science, 12(6), 473-477. 